ACCEPTED MANUSCRIPT

\title{
Noise spectroscopy of tunable nanoconstrictions: molecule-free and molecule-modified
}

To cite this article before publication: Volodymyr Handziuk et al 2018 Nanotechnology in press https://doi.org/10.1088/1361-6528/aad0b7

\section{Manuscript version: Accepted Manuscript}

Accepted Manuscript is "the version of the article accepted for publication including all changes made as a result of the peer review process, and which may also include the addition to the article by IOP Publishing of a header, an article ID, a cover sheet and/or an 'Accepted

Manuscript' watermark, but excluding any other editing, typesetting or other changes made by IOP Publishing and/or its licensors"

This Accepted Manuscript is @ 2018 IOP Publishing Ltd.

During the embargo period (the 12 month period from the publication of the Version of Record of this article), the Accepted Manuscript is fully protected by copyright and cannot be reused or reposted elsewhere.

As the Version of Record of this article is going to be / has been published on a subscription basis, this Accepted Manuscript is available for reuse under a CC BY-NC-ND 3.0 licence after the 12 month embargo period.

After the embargo period, everyone is permitted to use copy and redistribute this article for non-commercial purposes only, provided that they adhere to all the terms of the licence https://creativecommons.org/licences/by-nc-nd/3.0

Although reasonable endeavours have been taken to obtain all necessary permissions from third parties to include their copyrighted content within this article, their full citation and copyright line may not be present in this Accepted Manuscript version. Before using any content from this article, please refer to the Version of Record on IOPscience once published for full citation and copyright details, as permissions will likely be required. All third party content is fully copyright protected, unless specifically stated otherwise in the figure caption in the Version of Record.

View the article online for updates and enhancements. 


\title{
Noise Spectroscopy of Tunable Nanoconstrictions: Molecule-Free and
}

\section{Molecule-Modified}

\author{
Volodymyr Handziuk ${ }^{l}$, Ferdinand Gasparyan, ${ }^{1,2}$ Lode K. J. Vandamme, ${ }^{3}$ Maristella Coppola, ${ }^{1}$ \\ Viktor Sydoruk ${ }^{1+}$, Mykhailo Petrychuk, ${ }^{1,4}$ Dirk Mayer ${ }^{l}$ and Svetlana Vitusevich, ${ }^{1 *}$
}

${ }^{1}$ Bioelectronics (ICS-8), Forschungszentrum Jülich GmbH, 52425 Jülich, Germany

${ }^{2}$ Yerevan State University, 0025 Yerevan, Armenia

${ }^{3}$ Eindhoven University of Technology, 5600MB Eindhoven, Netherlands

${ }^{4}$ Faculty of Radiophysics, Electronics and Computer Syśstems, Taras Shevchenko National University of Kyiv, 03127 Kyiv, Ukraine

Keywords: transport regimes, nanojunctions, tunable nanoconstrictions, noise spectroscopy

${ }^{\dagger}$ Current institute: Institute of Bio- and Geosciences (IBG-2)

" Corresponding author Email address: s.vitusevich@,fz-juelich.de 


\begin{abstract}
Devices with metallic nanoconstrictions functionalized by organic molecules are promising candidates for the role of functional devices in molecular electronics. However, to the moment little is known about transport and noise properties of nanoconstriction devices of such kind. In this paper, transport properties of bare-gold and molecule-containing tunable cross-section nanoconstrictions are studied using low-frequency noise spectroscopy. Normalized noise power spectral density $S_{I} / I^{2}$ dependencies are analyzed for a wide range of sample resistances $R$ from $10 \mathrm{Ohm}$ to $10 \mathrm{MOhm}$. Peculiarities and physical background of the flicker noise behavior in the low-bias regime are studied. It is shown that modification of sample surface with benzene-1,4dithiol (BDT) molecules results in a decrease of the normalized flicker noise spectral density level in the ballistic regime of sample conductance. The characteristic power dependence of normalized noise power spectral density as a function of system resistance are revealed. Models describing noise behavior for bare gold and BDT modified samples are developed and compared with the experimental data for three transport regimes: diffusive, ballistic and tunneling. Parameters extracted from models by fitting are used for characterization of nanoconstriction devices.
\end{abstract}

\title{
1. Introduction
}

Nowadays the sizes of functional elements of electronic circuits have already reached nanometer scale. As the sizes of electronic devices continue decreasing, knowledge about the electron transport in one-dimensional atomic-sized channels became very important. Further downscaling will lead to utilizing of atomic and molecular complexes or even single molecules in electrical circuits. Understanding of fundamental mechanisms of the charge transport in metal-molecule systems and further control over their transport properties are important for the development of sub-nanometer functional devices. 
To design molecular electronics circuits metal-molecule junctions have to be studied, since their electrical properties are extremely sensitive to external influences and binding geometry. The mechanisms of charge transport and low-frequency noise behavior in thin metal films and nanowires as well as in discontinuous metal films have been the objects of numerous investigations over the course of several decades. ${ }^{1-16}$ However, transport and noise measurements of molecular systems involving metal-molecule junctions are still challenging. It was shown that some thiolterminated molecular junctions can exhibit very high random telegraph signal (RTS) noise levels. ${ }^{17,18}$ Such noise behavior was explained by vibrations, breaking-and reestablishing of the bonds between the molecule and metal electrodes. In the cases of more stable metal-moleculemetal systems (without bond breakings) it was revealed that RTS noise originates from the current induced reconfigurations of the molecule. ${ }^{19} \mathrm{On}$ the other hand flicker noise component is important and has to be analyzed. It may have relatively high amplitude and compete with RTS noise component, therefore $1 / \mathrm{f}$ noise has to be analyzed in molecule containing junctions and compared with the case of molecule-free junctions. Noise fluctuations can provide very useful information about strengths of molecular and atomic bindings as well as mechanisms of transport formation in the structures. Not only dynamical properties of the molecules themselves, but also the properties of the interface between molecules and electrical contacts have to be understood to utilize successfully the molecules and/or molecular layers in molecular electronics. In the case of molecule free metallic nanojunctions it was found that the normalized noise power spectral density $S_{I} / I^{2}$ has a power dependence on the resistance: ${ }^{15}$

$$
\frac{S_{I}}{I^{2}} \propto R^{m}
$$

Here $S_{I}$ is the current noise power spectral density (PSD), $m$ is the exponent. It was shown that the magnitude of the exponent $m$ is equal to 3 for the diffusive regime and for the higher resistance region, supposed to be ballistic one it was estimated as $m=1.5 .^{15}$ Metallic nanowires and 
nanoconstrictions with diameters of a few nanometers are sensitive to molecular adsorbates altering their surface charge density. In this respect, studies of metallic nanoconstriction systems modified by organic molecules in different transport regimes can provide useful information for developing of nanoscale functional devices with predefined properties. However, quantitative studies of the flicker noise behavior in devices with tunable metallic nanoconstrictions, modified by a molecular layer has not been reported yet.

In this work we present results of the flicker noise investigation on gold nanoconstrictions with bare surface and modified by benzene-1, 4-dithiol (BDT) molecules for a wide range of resistances from $10 \mathrm{Ohm}$ to $10 \mathrm{MOhm}$. In contrast to Ref. 15 we analyzed not only diffusive, but also ballistic and tunneling regimes in the sample and considered the influence of a molecular layer on noise properties of the system. Moreover, nanoconstrictions modified with BDT show different flicker noise behavior compared with bare ones. We address this effect to the influence of the molecular layer to overall conductance of the system as well as to the confinement of the configurational freedom of the gold atoms by stabilizing the constriction with the covalently bound BDT molecules.

\section{Experimental Methods}

Fabrication Procedure: Rectangular shape stainless steel pieces (width $=1 \mathrm{~cm}$, length $=5 \mathrm{~cm}$, thickness $=0.15 \mathrm{~mm}$ ) were used as substrates for our samples. At first, substrates were cleaned using organic solvents (acetone and then isopropanol). Two layers of polyimide (PI2611) were then subsequently spin-coated onto substrates to create a passivation. Each coating step was followed by heating up to 350 degrees in a furnace in order to harden the polyimide film. Then we spin-coated a stack of e-beam sensitive polymers (PMMA 669.04 and PMMA 679.04) above the passivation layer and performed patterning of our nanostructures by means of e-beam lithography. 
Resistance Tuning Procedure: We used our homemade setup to tune the cross-section of the suspended nanoconstriction in a precise way using a three-point mechanical bending system (pushing rod and two counter supports), controlled by a PC. The schematic of the mechanically controllable break junction setup is shown in Figure 1B. Horizontal displacement between electrodes $(\Delta x)$ is related to vertical shift of the pushing $\operatorname{rod}(\Delta \overline{)})$ via the attenuation factor $\alpha=\Delta x / \Delta=6 u t / L^{2}$, which is defined by the setup geometry. ${ }^{20}$ Here $u$ is the width of the underetched bridge, $t$ is the thickness of the substrate and $L$ is the distance between two counter supports. In our case, this parameter is $\alpha \approx 3 \times 10^{-7}$, which means that varying the position of pushing 
rod in micrometer scale allows us to tune the horizontal displacement in sub-angstrom range. Typical junction conductance dependence on the displacement of the pushing rod (breaking curve), measured at a constant voltage $V=20 \mathrm{mV}$ and conductance histogram are shown in Figure 2.
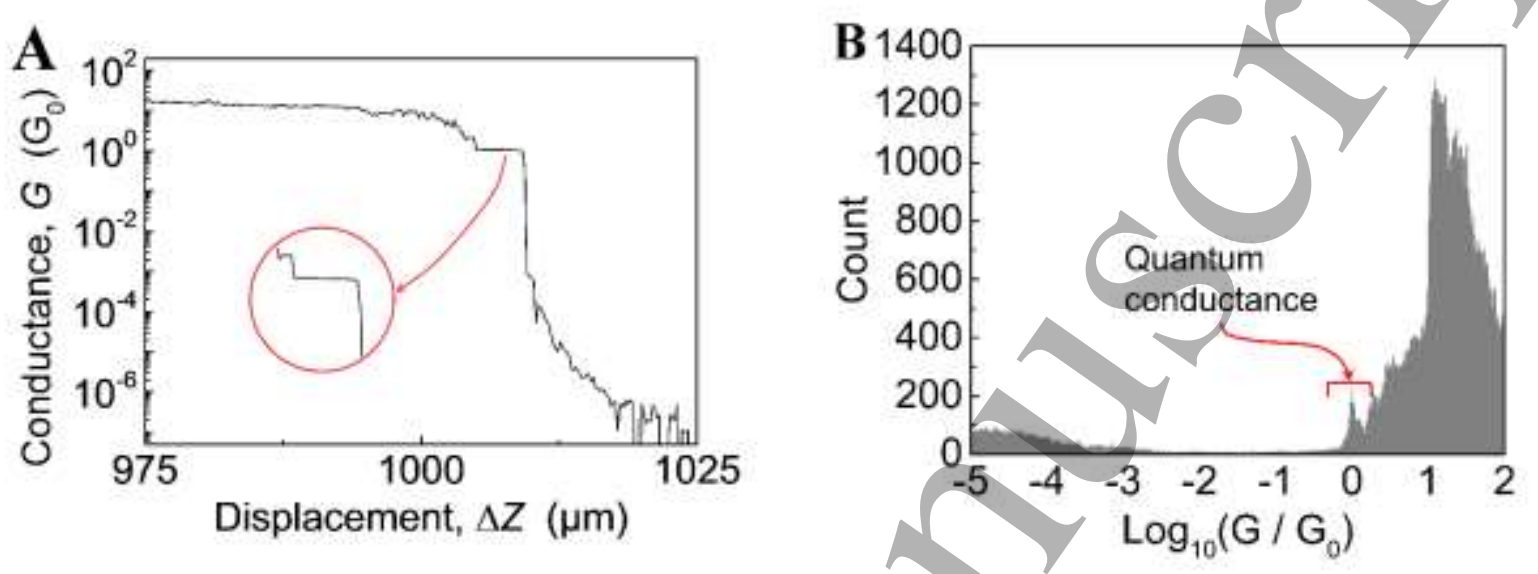

Figure 2. A) Typical conductance change of the sample with nanoconstriction as a function of the displacement of pushing rod and B) conductance histogram, measured on a bare gold sample.

Several quantum plateau (multiples of the quantum conductance) can be clearly resolved, which indicates good quality of fabricated samples.

Functionalization Procedure: Benzene-1,4-dithiol powder (99\% GC) was purchased from Sigma Aldrich. BDT (7.2 mg) was dissolved in ethanol $(5 \mathrm{ml})$ to get a $10^{-2} \mathrm{M}$ stock solution. Functionalization procedure was performed as follows. First samples with tunable gold nanoconstrictions were cleaned by rinsing with ethanol for 2 minutes. Then $200 \mu$ l of BDT solution $\left(10^{-4} \mathrm{M}\right.$, obtained by dilution of the stock solution) were applied to the nanoconstriction region of the samples by drop-casting. After 2 hours samples were rinsed in ethanol for one minute in order to remove the excess amount of BDT molecules that didn't bind gold covalently. Typical breaking curve and conductance histogram for the sample, modified with BDT is shown in Figure 3A and Figure 3B, respectively. The BDT conductance peak value is obtained by fitting the conductance 

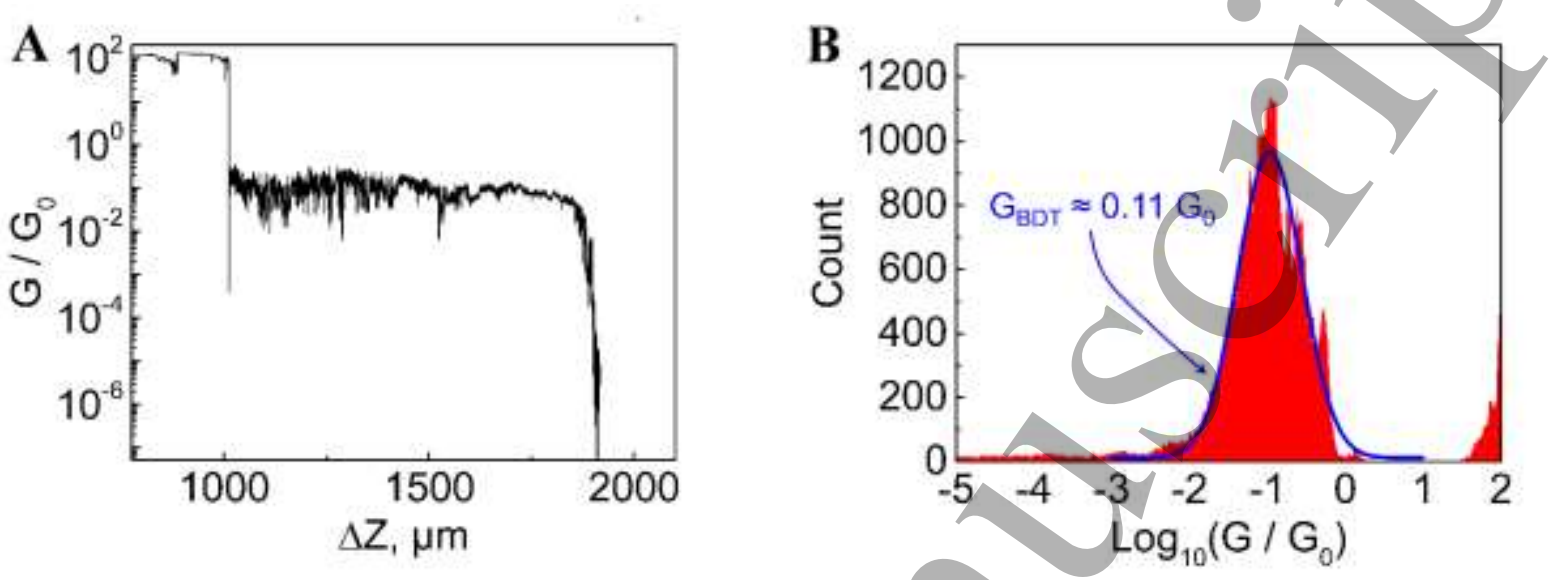

Figure 3. A) Typical breaking curve of the tunable nanoconstriction, modified with BDT molecules and B) the corresponding conductance histogram.

Noise Measurements: Constant voltage was applied to the serial connection of the sample and high precision load resistor $R_{\text {load }}=5000 \mathrm{Ohm}$ using lead-acid battery. Current fluctuations at the terminals of the junction were amplified by a cascade of ultra-low noise homemade preamplifier and commercial Stanford SR560 low noise amplifier. The first amplification step is very important, because it defines the accuracy of the data determination and therefore it should be optimized to obtain the lowest possible instrumental noise. Our preamplifier $(24 \mathrm{~dB})$ at $100 \mathrm{~Hz}$ has a level of intrinsic noise of $2.2 \times 10^{-18} \mathrm{~V}^{2} \mathrm{~Hz}^{-1}$, which is one order of magnitude lower than the noise level $\left(2.4 \times 10^{-17} \mathrm{~V}^{2} \mathrm{~Hz}^{-1}\right)$ of the commercial amplifier used. The amplified noise signal was then registered by a dynamic parameter analyzer HP35670A. Finally, recorded noise spectra were transferred via GPIB interface to a PC. A full description of our measurement setup can be found in Refs. 8, 23. 
Transport and noise characteristics were measured at a room temperature (297 K) under a high vacuum $\left(10^{-6} \mathrm{mbar}\right)$ in an electrically shielded environment. Noise spectra were studied at different resistances in the low-bias regime at a constant voltage $V=20 \mathrm{mV}$ applied. The measurements were repeated on 10 nanoconstriction devices. Each of the studied BDT-modified samples demonstrates a molecular conductance peak (see Figure 3 ) at $G_{\mathrm{BDT}} \approx 0.11 G_{0}$. In most cases normalized flicker noise level was approximately 6 times lower for BDT modified samples compared to bare gold nanoconstrictions at resistances in the range from $\left(4 G_{0}\right)^{-1}$ to $\mathrm{G}_{0}{ }^{-1}$. The results show reproducible conductance and flicker noise behavior. Estimated parasitic capacitance of the cable connection was $C=300 \mathrm{pF}$, resulting in the minimal cutoff frequency $f_{\text {cutoff }}=106 \mathrm{kHz}$ in the configuration of our setup. ${ }^{8}$ This value is far beyond the investigated frequency range $(10 \mathrm{~Hz}-1$ $\mathrm{kHz}$ ), thus the parasitic capacitance had no significant influence on the measurement results. Additionally, cut-off correction and subtraction of the thermal noise component were performed for the noise spectra before the analysis.

\section{Results and Discussion}

We used a noise spectroscopy technique to characterize sample transport properties in different regimes. Low-frequency noise spectra $(10 \mathrm{~Hz}-1 \mathrm{kHz})$ were measured over a wide range of system resistances, including regions before and after breaking of the constriction. Altering the resistance of the system by reducing the nanoconstriction area leads to changes in the nature of its dominant transport mechanism from diffusive at low resistances, ballistic at intermediate $R$, followed by quantum tunneling in the case of a broken nanoconstriction. Before each measurement, resistance of the sample was stabilized using a program-controlled feedback system, developed in house. Experiments were performed for bare gold nanoconstrictions as well as for those, modified with BDT molecules. 
Typical noise spectra are shown in Figure 4. Flicker noise is the dominant component of the noise spectra in the investigated range of resistances from $10 \mathrm{Ohm}$ to $10 \mathrm{MOhm}$. The normalized current noise power spectral density (at a frequency of $100 \mathrm{~Hz}$ ) dependencies on the system resistance are shown in Figure 5. Black points indicate the data obtained for bare gold nanoconstriction, while red points reflect data corresponding to the nanoconstriction modified with BDT molecules.

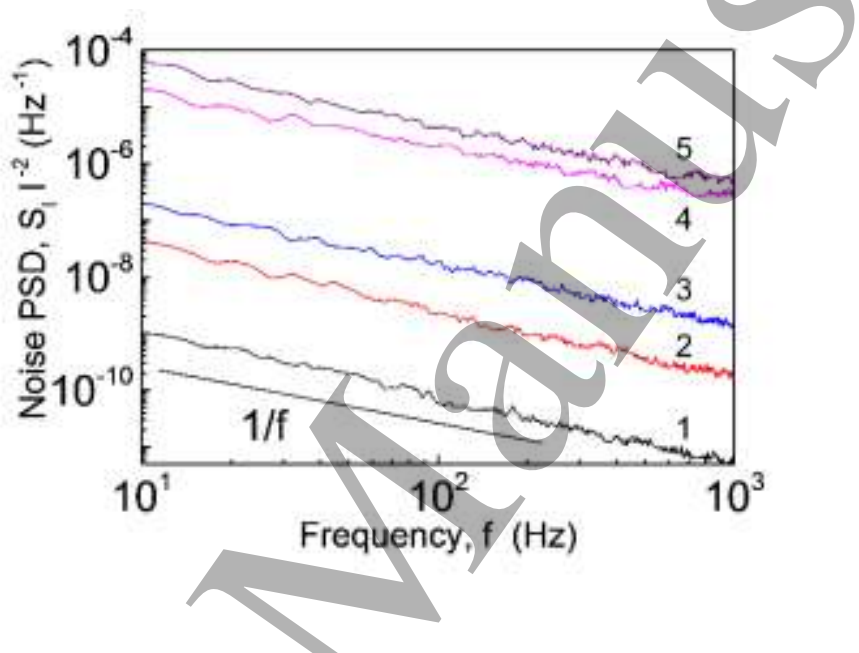

Figure 4. Normalized noise power spectral density curves before (1-3) and after $(4,5)$ the breaking of a nanoconstriction without molecules. The selected spectra correspond to the following values of the resistance $R: 1-0.36 \mathrm{kOhm} ; 2-0.77 \mathrm{kOhm} ; 3-2.02 \mathrm{kOhm} ; 4-223.00 \mathrm{kOhm} ; 5-642.60 \mathrm{kOhm}$, respectively.

With narrowing of the nanoconstriction due to the bending process and subsequent growth of its resistance, we observe an increase in the relative flicker noise level which has a power dependence on the resistance described by Equation (1). At low resistances $(R<300 \mathrm{Ohm})$ the exponent has the value $m=2$. Starting from the resistance $R \approx 3000 \mathrm{Ohm}$ the value of $m$ decreases with the resistance increase. We address the behavior of the magnitude $m$ to changes in transport properties of the sample due to shrinking the characteristic size of the nanoconstriction down to the mean free path of charge carriers $\left(\lambda_{\mathrm{e}} \approx 4 \mathrm{~nm}\right.$ in gold at room temperature $\left.{ }^{24}\right)$. 

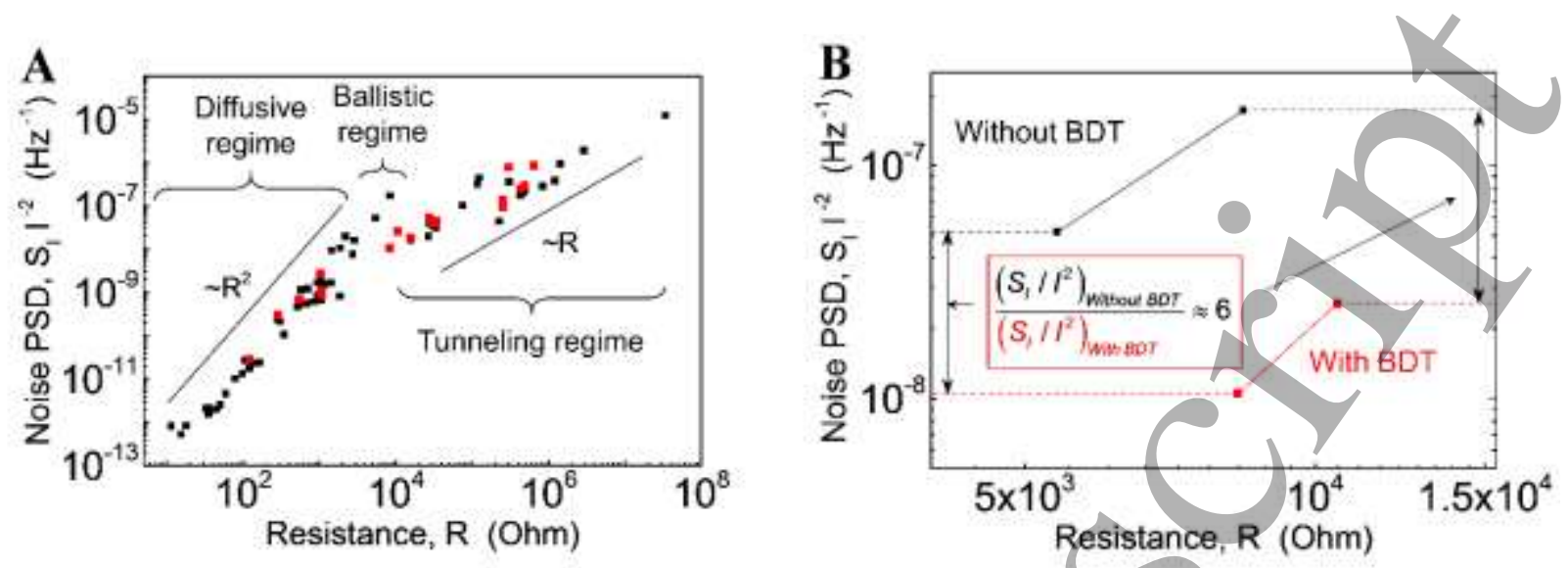

Figure 5. A) Normalized flicker noise power spectral density, obtained at $100 \mathrm{~Hz}$ as a function of the nanoconstriction resistance. Black squares show data obtained a bare nanojunction, red ones correspond to the case of the presence of BDT molecules. B) Enlarged section of the transition from diffusive to tunneling regime, including ballistic region.

\subsection{Noise Behavior in Diffusive Regime}

It was previously shown, ${ }^{25}$ that gold nanocontacts have stable mechanical and electronic structure at resistances lower than $R=\left(4 G_{0}\right)^{-1}$. At low biases applied and relatively low resistance values, the electric transport in our system can be considered as diffusive. The flicker current noise is a result of $1 / f$ fluctuations in conductivity. For simplicity of resistance and noise calculations we will consider our system as a $2 \mathrm{D}$ resistor. For the $2 \mathrm{D}$ case the power dissipation can be calculated using following equations:

$$
\begin{aligned}
& R I^{2}=\int_{\text {Area }} R_{s h} J^{2} d A ; \\
& G V^{2}=\int_{\text {Area }} \frac{E^{2} d A}{R_{s h}} .
\end{aligned}
$$


Here $R_{s h},[\mathrm{Ohm}]$ is the sheet resistance, $J,[\mathrm{~A} / \mathrm{cm}]$ is $2 \mathrm{D}$ current density, $G[\mathrm{~S}]$ is the conductance, $E,[\mathrm{~V} / \mathrm{cm}]$ is the electric field strength and $d A$ is an elementary area. As it is shown in Refs. 2631 , spectral density of the flicker noise $S_{R}$ is proportional to $\int J^{4}$ :

$$
S_{R}=\frac{C_{u s} R_{s h}^{2}}{f I^{4}} \int_{\text {Area }} J^{4} d A
$$

with the $1 / f$ parameter $C_{u s}\left[\mathrm{~cm}^{2}\right]$ inversely proportional to gold layer thickness and defined for a unit square material of $1 \mathrm{~cm}^{2}$ submitted to homogeneous field. It turns out that $C_{u s} / R_{s h} \approx 5 \times 10^{-21}$ $\mathrm{cm}^{2} / \Omega$ for uniform gold layers and poly-silicon of different thickness. ${ }^{31}$ The precise calculation of the integrals in $2 \mathrm{D}$ is often done by solving finite-difference equations $\mathrm{s}^{32,33}$ or by conformal mapping. ${ }^{34,35}$ An analytical solution based on approximated equipotentials has an advantage, especially for the relative noise $S_{R} / R^{2}$, because errors in $S_{R}$ and $R$ are partially cancelled out. ${ }^{26}$

Top view of the sample is shown in Figure 6A, and its schematic view - in Figure 6B. For ease of calculations, we arbitrarily divide the nanoconstriction region in two parts: trapezoidal access part, which does not change during the tuning process and tunable inner part (narrow neck). First we consider the noise behavior of the access parts.

\subsubsection{The resistance and noise estimation of trapezoidal access parts}

The calculation of resistance and $1 / f$ noise of trapezoidal access part are based on simplified equipotentials. The equipotentials are assumed to be straight lines perpendicular to the $x$-axis (see Figure 6B). In the model proposed, resistance and noise of the trapezoidal access parts are considered to remain constant/during the bending. Main contribution to resistance and noise comes from the inner part of the nanoconstriction due to a reduction of its effective width. 

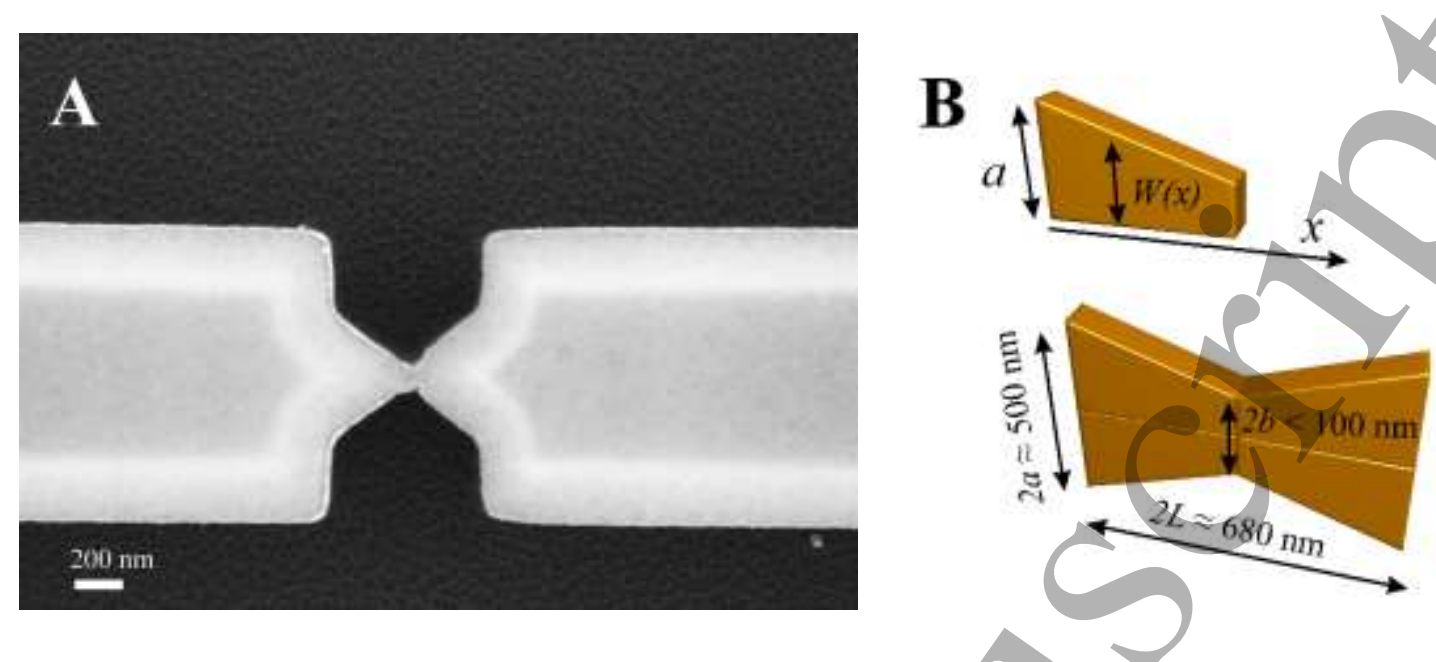

Figure 6. A) Typical SEM micrograph of the top view of the sample. B) Model of the access part. Top: Geometrical model of the trapezoidal access part to the narrow neck as a quarter of the complete access part for the calculations of resistance and noise. Bottom: Trapezoidal access parts with the dimensions shown in the figure, without a narrow neck part.

The sketch of trapezoidal shaped access part without a narrow neck is shown in the Figure 6B with the dimensions along $x$ and $y$ axes $2 L$ and $2 a$, respectively. Due to a symmetry of the system, resistance $R$ and noise $S_{R}$ calculations are performed on a quarter part (Figure 6B, on the top) and then the overall access resistance and noise are calculated by an appropriate addition of four equal parts. Total resistance and noise of the system are denoted by $R_{\otimes}, S_{R_{\circledast}}$ and $S_{R_{\circledast}} / R_{\otimes}^{2}$ respectively.

Considering the geometry of the system, the width of the structure $W(\mathrm{x})$ at the position $x$ can be described by the following equation:

$$
W(x)=-\frac{a-b}{L} x+a .
$$

The current density at the point $x$ is $J(x)=I / W(x)=I /(x(-(a-b) / L)+a)$. The resistance of a quarter access part of the nanoconstriction can be found using Equation (2): 


$$
R=R_{s h} \frac{L}{a-b} \ln \frac{a}{b}
$$

The resistance noise of a trapezoidal quadrant part, calculated using Equation (3) and relative resistance noise are given by:

$$
S_{R}=\frac{C_{u s} R_{s h}^{2}}{2 f} \frac{L}{a^{2}} \frac{(\mathrm{a} / \mathrm{b})^{2}-1}{a-b} \Rightarrow \frac{S_{R}}{R^{2}}=\frac{C_{u s}}{2 f} \frac{a-b}{L a^{2}} \frac{(\mathrm{a} / \mathrm{b})^{2}-1}{(\ln \mathrm{a} / \mathrm{b})^{2}}
$$

The complete access part of consists of four equal trapezoidal quadrants, two of which are connected in series and two - in parallel. Therefore, the total resistance, $R_{\otimes}$, resistance noise $S_{R_{\otimes}}$ and relative noise $S_{R_{\circledast}} / R_{\otimes}^{2}$ of the access part are given by:

$$
\begin{aligned}
& R_{\otimes}=2 R / 2=R=R_{s h} \frac{L}{a-b} \ln \frac{a}{b} \\
& S_{R_{\otimes}}=\frac{1}{4} S_{R} \Rightarrow S_{R_{\circledast}}=\frac{1}{4} \frac{C_{u s} R_{s h}^{2}}{2 f} \frac{L}{a^{2}} \frac{(\mathrm{a} / \mathrm{b})^{2}-1}{a-b} \\
& \frac{S_{R_{\circledast}}}{R_{\otimes}^{2}}=\frac{1}{4} \frac{S_{R}}{R^{2}} \Rightarrow \frac{S_{R_{\circledast}}}{R_{\circledast}^{2}}=\frac{C_{u s}}{8 f} \frac{a-b}{L a^{2}} \frac{(\mathrm{a} / \mathrm{b})^{2}-1}{(\ln \mathrm{a} / \mathrm{b})^{2}}
\end{aligned}
$$

Using Equation (9) and taking into account parameters of the real sample we can estimate the value of the relative noise level of the access parts: $S_{R} /\left.R^{2}\right|_{\text {access }} \approx 2 \times 10^{-14}$ at $100 \mathrm{~Hz}$. Now we consider the noise behavior of the tunable inner part.

\subsubsection{Resistance and noise estimation of the narrow neck}

Bending of the sample results in an elongation of the narrow neck, its plastic deformation and subsequent breakage. Resistance and noise estimations are based on the current crowding due to a decreasing of the cross-section of the nanoconstriction, treated as a bending sector (shown in Figure 7 with $2 b=W$, the characteristic radius $r_{1}$ a small fraction of $W: r_{1}=p W ; p \ll 1$ and $r_{2}=$ 
$\left.r_{1}+W_{e f f} \Rightarrow r_{2} / r_{1}=1+W_{e f f} / p W\right)$. Width and length of the tunable inner part before damage are equal to $W=2 b$.
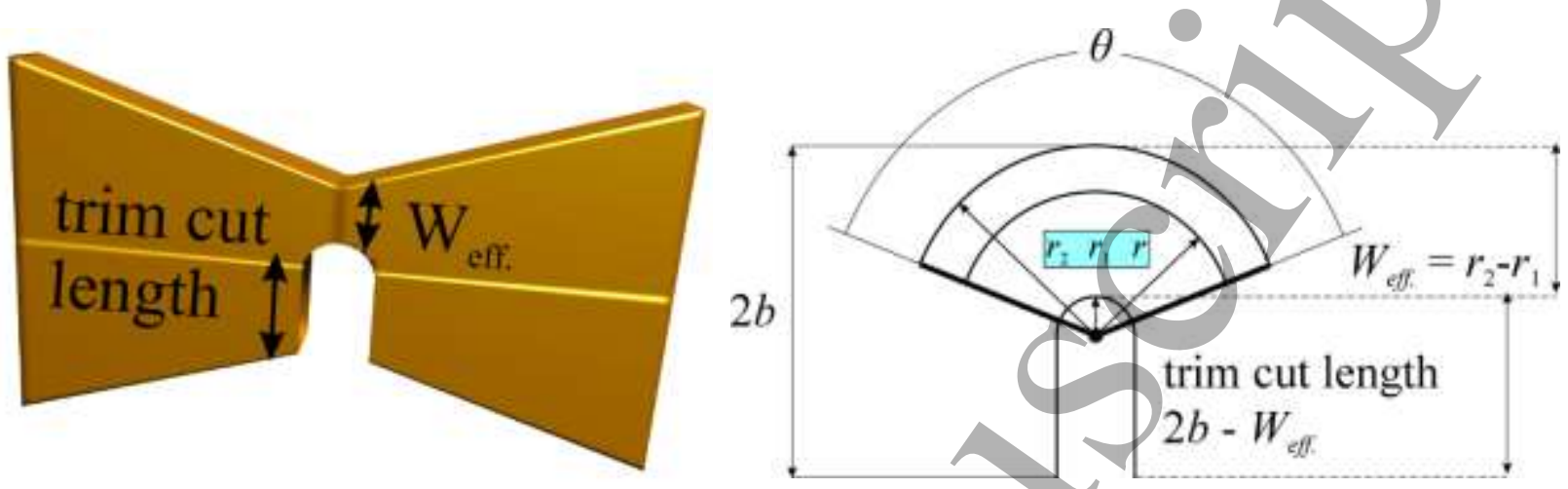

Figure 7. Sketch of the damaged nanoconstriction (left-hand side) and bending sector around the trim-cut (right-hand side), caused by the damage, showing the circular current lines between radii $r_{1}$ and $r_{2}$. The bending of current lines due to a trim-cut is shown over an angle $\theta<\pi$. Assumed equipotentials are drawn as thick lines.

The analytical solution for resistance and noise is based on approximated current lines around the trim-cut, caused by damage as shown in Figure 7. In this region, the electric field is higher and is the highest for the radius $r$ close to the smallest rounding-off radius $r_{1}$. The biggest radius of the sector is equal to $r_{2}=r_{1}+W_{\text {eff }}$ and hence, $r_{2} / r_{1}=1+W_{\text {eff }} / r_{1}$. The electrical field strength and current density within the sector with radii $r$ and $r+d r$ equal the voltage drop $V$ over the assumed equipotential lines: $E(r)=V /(\theta r)$ and $J(r)=V /\left(R_{s h} r \theta\right) \cdot{ }^{35}$ Calculations, performed using Equation (2) and (3), give following results for the resistance and resistance noise:

$$
\begin{aligned}
& R=\frac{\theta R_{\text {sh }}}{\ln \left(1+W_{\text {eff }} / r_{1}\right)} \\
& S_{R}=\frac{R_{s h}^{2} C_{u s} \theta}{2 f\left(\ln \left(1+W_{\text {eff }} / r_{1}\right)\right)^{4}}\left[\frac{1}{r_{1}^{2}}\right]\left[\frac{\left(W_{\text {eff }} / r_{1}\right)^{2}+2\left(W_{\text {eff }} / r_{1}\right)}{\left(\left(W_{\text {eff }} / r_{1}\right)+1\right)^{2}}\right] \propto R^{4} \text { for } \frac{r_{1}}{W_{\text {eff }}+r_{1}}<1
\end{aligned}
$$


To find the dependence of the relative current noise level on the system resistance, we use the general relation between relative values of current, conductance and resistance noise levels: $:^{36,37}$

$$
\frac{S_{I}}{I^{2}}=\frac{S_{G}}{G^{2}}=\frac{S_{R}}{R^{2}},
$$

where $S_{\mathrm{G}} / G^{2}$ is the relative conductance noise level. Using Equation (11) and (12) we can obtain following expression for the relative current noise:

$$
\frac{S_{I}}{I^{2}}=\frac{S_{R}}{R^{2}}=\frac{C_{u s}}{2 \theta f\left(\ln \left(1+\frac{W_{e f f}}{r_{1}}\right)\right)^{2}}\left[\frac{1}{r_{1}^{2}}\right]\left[\frac{\left(\frac{W_{e f f}}{r_{1}}\right)^{2}+2 \frac{W_{\text {eff }}}{r_{1}}}{\left(\frac{W_{\text {eff }}}{r_{1}}+1\right)^{2}}\right] \Rightarrow \begin{gathered}
\propto \mathbf{R}^{2} \text { for } \frac{r_{1}}{W_{e f f}+r_{1}}<1 \\
\propto \mathbf{R} \text { for } \frac{W_{\text {eff }}+r_{1}}{r_{1}} \downarrow 1
\end{gathered}
$$

As can be seen from Equation (13), there are two cases of the relative flicker noise level scaling with the sample resistance depending on the relation between the values of $r_{1}$ and $W_{\text {eff. }}$. In our case we registered the dependence with the power $m=2$. The results for the other transport regimes are analyzed and summarized below.

\subsection{Noise Behavior in Ballistic Regime}

When characteristic sizes of the nanoconstriction are comparable with the mean free path of electrons $\lambda_{\mathrm{e}}$, the electrical transport in the system changes. At this point no scattering events take place in the narrowest part of the nanojunction in a passage. At resistances corresponding to the ballistic regime only a few gold atoms are connecting two bulky electrodes. In this case the resistance of the system is mainly determined by the quantum resistance of the interconnecting atoms, forming a wire.

\subsubsection{Model for Bare-Gold Nanoconstrictions}

We assume that elongation of the suspended nanojunction in its narrowest part due to the bending causes a rearrangement of gold atoms, keeping constant the area of the nanoconstriction $A_{\text {consstr: }}=c \times d=$ const (see Figure 8). 

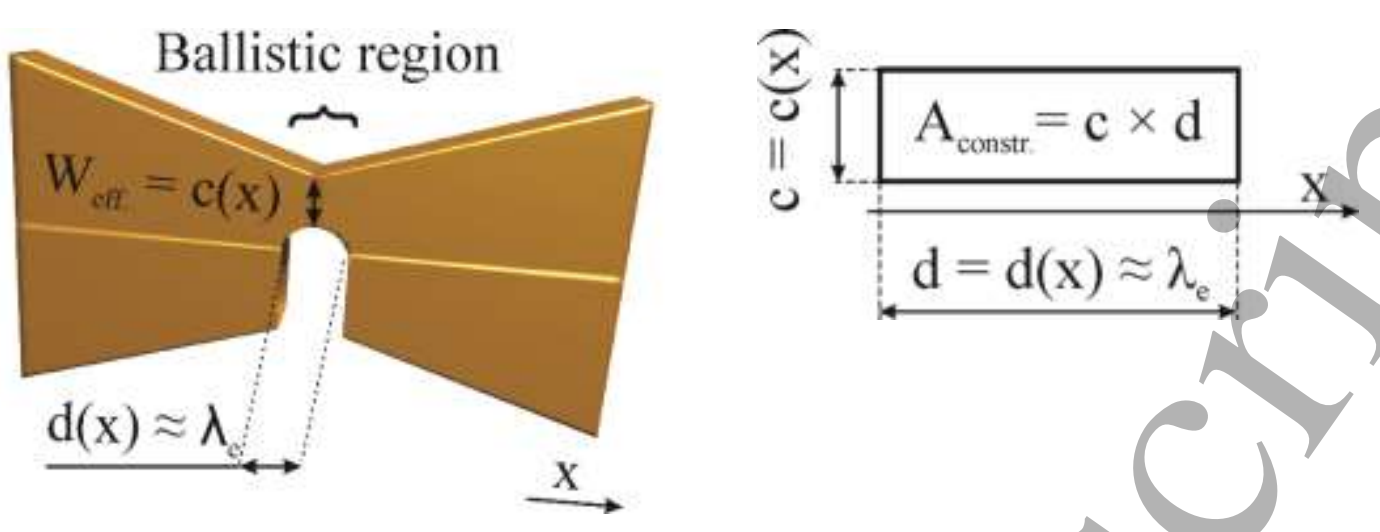

Figure 8. Schematic of the nanoconstriction inner part in the ballistic regime of conductance

The resistance of the sample in ballistic conductance regime is defined by Sharvin resistance:

$$
R_{S}=\frac{4 \rho \lambda_{e}}{3 \pi r_{c}^{2}}
$$

where $\rho$ is the resistivity of the sample material and $r_{c}=c / 2$ is the ballistic contact radius. The resistivity can be written as $\rho=m_{e} v_{F} /\left(n e^{2} \lambda_{e}\right),{ }^{38}$ where $m_{e}$ is the electron mass, $v_{F}$ is the Fermi velocity, $n$ is the free electron density and $e$ is the electron charge. Therefore, from Equation (14) we can obtain following expression for the resistance:

$$
R_{S}=\frac{\left(\frac{4 m_{e} v_{F}}{3 \pi n e^{2}}\right)}{r_{c}^{2}}=\frac{C_{1}}{r_{c}^{2}},
$$

where constant $C_{1}=3.58 \times 10^{-16}\left[\mathrm{Ohm} \mathrm{m}^{2}\right]$ for gold. ${ }^{38}$

Dimensions of the elongated part (width $c$ and length $d$ ) change during the bending process. Consequently, they depend on the current value of the displacement between the electrodes $(x)$ :

$$
\begin{aligned}
& d=f(x)=d_{0}+x \\
& \mathrm{c}=f(x)=A_{\text {constr. }} / d(x)
\end{aligned}
$$


In this case, the resistance of the constriction is defined by the following expression:

$$
R_{S}=\frac{4 C_{1}}{c^{2}(x)}=\frac{d^{2}(x) 4 C_{1}}{A_{\text {constr. }}^{2}} \Rightarrow d=\sqrt{A_{\text {constr. }}^{2} \frac{R_{S}}{4 C_{1}}}
$$

The flicker noise component and its dependence on the sample resistance can be estimated using the Hooge formula:

$$
S_{I}=\frac{\alpha_{H} e I}{\tau f}
$$

where $S_{I}$ is the current noise spectral density, $\alpha_{H}$ is the dimensionless Hooge parameter and $\tau$ is the characteristic time. In the framework of our model, $\tau$ denotes the time, which electron spends in order to pass the distance between bulky electrodes separated by the ballistic region. In the quasiballistic regime, the velocity of electrons is determined by the thermal velocity:

$$
v_{T}=\sqrt{\frac{k T}{m_{e}}}
$$

where $v_{T}$ is the thermal velocity of electrons, $k$ is the Boltzmann constant, $T$ is the temperature and $m_{e}$ is the electron mass. Electrons motion from one electrode to another under the influence of the electric field can be described by the following equation:

$$
x(t)=x_{0}+v_{0} t+\frac{a_{e} t^{2}}{2}
$$

where $x_{0}=0$ is the initial position of an electron, $v_{0}=v_{T}$ is the speed of the electron at its initial position and $a_{e}$ is its acceleration in electric field. In our experiments, we apply a constant voltage to the sample:

$$
V=U_{0} ; I=U_{0} / R
$$

Therefore, the acceleration $a_{e}$ can be found using the expression for the electric force and Newton's law as: 


$$
\begin{gathered}
F=e E, E=U_{0} / d \Rightarrow a_{e}=\frac{F}{m_{e}}=\frac{e U_{0}}{m_{e} d} . \\
F=m_{e} a_{e}
\end{gathered}
$$

By solving Equation (20), considering that $x(t=\tau)=d$ we can obtain the expression for $\tau$.

$$
\tau=d \frac{m_{e}}{e U_{0}}\left(\sqrt{v_{T}^{2}+2 e U_{0} / m_{e}}-v_{T}\right)=C \cdot d ; C=\frac{m_{e}}{e U_{0}}\left(\sqrt{v_{T}^{2}+2 e U_{0} / m_{e}}-v_{T}\right) .
$$

Using Equation (18), (21) and (23), we can get the equation for the current noise spectral density:

$$
S_{I}=\frac{1}{f} \frac{2 \alpha_{H} e U_{0}}{A_{\text {constr. }} C \sqrt{1 / C_{1}}} R_{S}^{-3 / 2} \sim R_{S}^{-3 / 2}
$$

Using Equation (21) and (24) we can obtain following expression for the relative current noise:

$$
\frac{S_{I}}{I^{2}}=\frac{1}{f} \frac{2 \alpha_{H} e}{A_{\text {constr. }} C \sqrt{1 / C_{1}} U_{0}} R_{S}^{-1 / 2} \sim \sqrt{R_{S}} .
$$

Therefore, ballistic transport regime can be described by the square root dependence on the junction resistance. The experimental results are in a good agreement with theoretically predicted dependencies described by Equation (25). This fact allows to suggest that the flicker noise power dependence can be used as the characteristic parameter confirming the evidence of the ballistic transport in the system.

\subsubsection{Model considering the influence of molecular layer on noise properties of the system}

Usually, conductance of metal nanoconstrictions consists of bulk conductance and surface conductance. For very thin films and nanowires the contribution of the surface conductance is significantly increased. Therefore, the total conductance of the nanoconstriction can be strongly modified by organic adsorbates and the layer of molecules deposited onto the metal nanoconstriction can influence the transport and noise properties of the system:

$$
\begin{aligned}
& G=G_{\text {bulk }}+G_{\text {surface }} ; G_{\text {surafce }} \propto A_{\text {eff. }} . \\
& S_{G}=S_{G_{\text {bulk }}}+S_{G_{\text {sufface }}}
\end{aligned}
$$


Here $G_{b u l k}$ is the bulk gold conductance; $G_{\text {surafce }}$ is the surface conductance of gold modified by a molecular layer; $A_{\text {eff. }}$ denotes the effective area of a molecular layer, influencing the conductance of the system. Using the Equation (12) we can obtain following expression for the normalized noise level:

$\frac{S_{I}}{I^{2}}=\frac{S_{G}}{G^{2}}=\frac{S_{R}}{R^{2}}=\frac{S_{G_{\text {bulk }}}+S_{G_{\text {sufface }}}}{\left(G_{\text {bulk }}+G_{\text {surface }}\right)^{2}}$

At low resistance values, we have a situation where $G_{\text {surface }} \leq G_{\text {bulk }} \Leftrightarrow S_{G_{\text {sumface }}} \leq S_{G_{\text {bulk }}}$, i.e. molecular layer does not influence the noise behavior of the system $S_{I} / I^{2} \approx S_{G_{b u l k}} / G_{b u l k}^{2} \sim R^{m}$. This case is described above. At high values of the resistance, before the nanoconstriction breaks, we have $G_{\text {surface }} \geq G_{\text {bulk }} \Leftrightarrow S_{G_{\text {sufface }}} \geq S_{G_{\text {bulk }}}$. Consequently:

$\frac{S_{I}}{I^{2}} \approx \frac{S_{G_{\text {layer }}}}{G_{\text {layer }}^{2}} \propto \frac{1}{A_{\text {eff. }}} \propto R$

Thus, the evidence of the molecular layer in tunable gold nanoconstrictions can be confirmed by monitoring a change in the flicker noise behavior from $S_{I} / I^{2} \sim R^{2}$ to $S_{I} / I^{2} \sim R$ at relatively high resistances.

\subsection{Noise Behavior in Tunneling Regime}

After a complete breaking (electrodes are not connected neither by gold atoms nor by BDT molecules), current in the system is formed by the tunneling processes through a nanoscale gap. The origin of the flicker noise in this case is related to the scattering processes of the tunneling electrons on the electrodes. After the breaking, bare and molecule-modified junctions demonstrate the same level of normalized flicker noise (see Figure 5), because in this case molecules do not influence the transport mechanisms of the system any more. 
To estimate the relative noise level for the ballistic regime Hooge formula for flicker noise and the $\tau$ dependence on the sample resistance are used. Solving the equation of the electron motion in the electric field (Equation (20)) and taking the initial velocity $v_{0}=0$ we can obtain for $\tau$.

$$
\tau=d \sqrt{\frac{2 m_{e}}{e U_{0}}}
$$

where $\tau$ denotes the time, which electron spends to pass the distance between bulky electrodes separated by a nanogap and $d$ is the width of the nanogap. In the tunneling region electrical current exponentially depends on the distance between electrodes and expression for current can be represented as follows: $:^{39}$

$$
I_{D T} \sim \mathrm{A}_{\text {emitter }} \mathrm{e}^{-\beta d}
$$

where $\beta=2 \sqrt{2 m_{e} \Phi} / h$ is the constant, which depends on the metal work function; $A_{\text {emitter }}$ is the effective electrode emitting area. In our experiments we apply a constant voltage $\left(V=U_{0}\right)$ to the sample. Therefore, the resistance of the system is determined by:

$$
R=\frac{V}{I}=\frac{U_{0}}{I} \sim \frac{1}{A_{\text {emitter }}} U_{0} e^{\beta d} .
$$

Solving Equation (31) for $d$ we can obtain:

$$
d \sim\left(\ln \frac{R}{U_{0}}\right) / \beta \sqrt{\frac{2 m_{e}}{e U_{0}}}
$$

Using Equation (18) we can obtain for the flicker noise level:

$$
\frac{S_{I}}{I^{2}}=\frac{1}{f} \frac{\alpha_{H} e \beta A_{\text {emitter }}}{U_{0} \sqrt{\frac{2 m_{e}}{e U_{0}}}} \frac{R}{\ln \frac{R}{U_{0}}} \sim \frac{1}{f} \frac{R}{\ln R}
$$

As can be seen from Equation (33), noise power has a weaker dependence on the resistance ( $\left.S_{I} / I^{2} \sim R / \ln (R)\right)$ in the tunneling regime of sample conductance in comparison to diffusive 
region. The comparison of the experimental and modeling results are described below and summarized in Table 1.

\subsection{Comparison of Model and Experiment for Different Transport Regimes}

Experimental data are plotted in Figure 9 together with theoretically calculated dependencies for comparison. Black and red squares correspond to data, acquired for bare gold and modified with BDT samples, respectively. Curves, defined by the models for each of the considered transport regimes are drawn in lines. Parameters of the models, extracted by fitting of experimental results are described below.

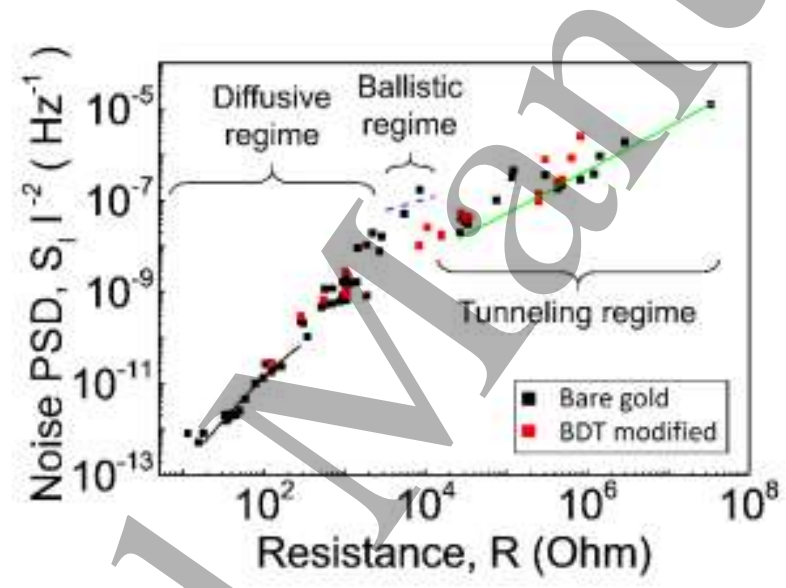

Figure 9. Comparison of experimental data from Figure 5 with theoretical dependencies obtained for different transport regimes. Black curve represents calculated data for the diffusive transport regime, blue one - for the ballistic transport regime, respectively. Green curve represents the results of simulations for the tunneling regime using the expression for tunneling transport.

For low resistance values $(R \leq 300 \mathrm{Ohm})$ in the diffusive transport regime, the simulation results of resistance and relative noise level (Equation (10) and (13)) are shown for: $\theta=160^{\circ}, r_{1}=3 \mathrm{~nm}$, $0.5 \leq W_{\text {eff }} / r_{1} \leq 500, R_{s h}=40 \mathrm{Ohm}$ and $C_{u s} / f=10^{-7} \mathrm{~nm}^{2} \mathrm{~Hz}^{-1}$ (black curve in Figure 9). Current noise 
of the access part $\left(S_{I} /\left.I^{2}\right|_{\text {access }}=S_{R} /\left.R^{2}\right|_{\text {access }} \approx 2 \times 10^{-14}\right.$ at $\left.100 \mathrm{~Hz}\right)$ is much lower than the noise, given by the narrow neck during the tuning process and therefore can be neglected. Angle $\theta$, as well as the effective width of the nanoconstriction $W_{\text {eff }}$ can be extracted by fitting. A model, based on a change of the relative noise level due to the geometry change of the inner part of the nanoconstriction, describes the behavior of noise well in the defined resistance range. At resistances higher than $R \geq 300 \mathrm{Ohm}$ the nanoconstriction shrinks in its dimensions rapidly with the increase of the resistance and at some point we can get a ballistic or one dimensional quantum channel. This leads to a transition region where we have a competitive impact of diffusive and ballistic regimes.

Model curve, describing normalized noise level in the ballistic transport regime is plotted in blue for the resistance range from $\left(4 G_{0}\right)^{-1}$ to $G_{0}^{-1}$. Proposed model allows estimation of the dimensionless Hooge parameter and the effective area of nanoconstriction, which demonstrates ballistic properties. The parameters for Hooge constant $\alpha_{H}=2.5 \times 10^{-5}$ and constriction area $A_{\text {constr. }}=2 \mathrm{~nm}^{2}$, extracted from the fitting of our experimental data are in a good agreement with physical considerations about the system and previously reported results. ${ }^{24,40}$ The model shows reliable values for the relative noise level. In the case of the presence of the molecular layer on the structure surface, decrease of the relative noise level is revealed. This effect can be characterized by the effective area $A_{\text {eff: }}$ (see Equations (27) and (28)) of the molecular layer, which contributes to the overall system conductance. At the resistances right above $R \approx 12900 \mathrm{Ohm}$, corresponding to the single atom conductance there is a drop down of the normalized flicker noise level, especially pronounced for the bare-gold samples. This effect occurs due to the sharp change of the transport regime from ballistic to tunneling one at higher resistances. 
Results of numerical calculations for tunneling regime $(R=25 \mathrm{kOhm} \div 10 \mathrm{MOhm})$ are plotted as green curve. Effective barrier height $\Phi$ and/or effective electron emitting area $\left(A_{\text {emitter }} \approx 20 \mathrm{~nm}^{2}\right)$ are estimated by fitting of the experimental data.

Table 1. Characteristic dependencies of the flicker noise in studied tunable gold nanoconstrictions

\begin{tabular}{lllll}
\hline \hline Dominant & Description & $\begin{array}{l}\text { Approximate } \\
\text { resistance } \\
\text { transport }\end{array}$ & $\begin{array}{l}\text { Characteristic } \\
\text { range }[\mathrm{Ohm}]\end{array}$ & $\begin{array}{l}\text { flicker nodel Parameters } \\
\text { behavior }\end{array}$
\end{tabular}

\begin{tabular}{ll|lll}
\hline Diffusive & Low-ohmic: bulk $\leq 300$ & $S_{I} / I^{2} \sim R^{2}$ & $W_{\text {eff. }}$ & Effective width of
\end{tabular} conductance properties

the nanoconstriction

$\theta \quad$ Angle characterizing current crowding in the nanoconstriction

$\begin{array}{ll}\text { Ballistic } & \text { Few gold atoms in } \\ \text { (BR1) } & \text { parallel: without }\end{array}$ BDT molecules

Ballistic Few gold atoms in 3000-12900 (BR2) parallel: with BDT molecules
$S_{I} / I^{2} \sim \sqrt{ } R$

$\alpha_{H}$

$A_{\text {constr: }}$

$S_{I} / I^{2} \sim R, \quad A_{\text {eff: }}$ lower amplitude than in BRl case

Tunneling $\quad>12900 \quad S_{I} / I^{2} \sim R / \ln R \quad \Phi$

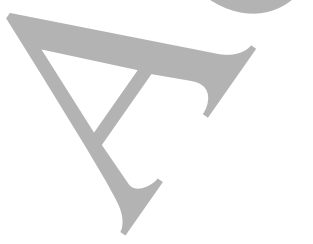


High ohmic: case

of broken

$A_{\text {emitter }}$

Effective electron

nanoconstriction

emitting area

To summarize, we developed models to explain the flicker noise behavior in tunable crosssection metallic nanoconstrictions in different transport regimes. Parameters extracted from the models by fitting describe change of the nanoconstriction geometry in a wide range of resistances as well as the effective area of a molecular film in the case of a molecule-modified system. Different transport regimes demonstrate characteristic dependencies of the flicker noise power (see Table 1). Therefore, data obtained by noise spectroscopy can be used for identification of the dominant transport regime in tunable nanoconstrictions.

\section{Conclusion}

Samples with tunable nanoconstrictions were fabricated using advanced cleanroom technology. Transport and noise properties of bare gold and molecule-modified nanoconstrictions were studied during the resistance tuning process for a wide range of sample resistances from $10 \mathrm{Ohm}$ to $10 \mathrm{MOhm}$. Flicker noise dependencies on the system resistance before and after breaking of the nanoconstriction are analyzed for diffusive, ballistic and tunneling regimes. The normalized flicker noise demonstrates a specific to the transport regime power dependence on the system resistance $S_{I} / I^{2} \sim R^{m}$. At low resistances before the nanoconstriction breaks, normalized noise power scales proportionally to $R^{2}$, while at higher resistances the magnitude of the exponent $m$ decreases for bare gold as $S_{I} / I^{2} \sim \sqrt{ } R$; for molecule-modified nanoconstrictions as $S_{I} / I^{2} \sim R$. Modification of the sample surface by BDT molecules results in a decrease of the normalized flicker noise level in comparison with bare-gold samples. The results are explained taking into account the molecular layer influence on the overall sample conductance at relatively high resistances and the sample 
stabilization by covalently bound molecules. Models are developed to explain the peculiarities and physical background of the noise behavior in different transport regimes of the sample conductance and summarized in Table 1. Developed models describe experimental data well in diffusive, ballistic and tunneling regimes. Parameters, extracted by fitting the experimental data are useful for characterization of such kind devices.

\section{Acknowledgements}

V.H. and F.G. contributed equally to this work. F. Gasparyan greatly appreciates the support from the German Academic Exchange Service (DAAD) in the form of a research grant. This work was supported by the RA MES State Committee of Science, in the framework of the research Project No. 15T-1C 279. Nanoconstriction devices were fabricated using the Helmholtz Nanoelectronic Facility of Forschungszentrum Jülich.

References:

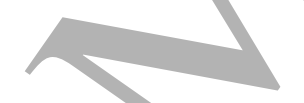

(1) Hooge, F. N. Discussion of Recent Experiments on 1/f Noise. Physica 1972, 60 (1), 130144.

(2) Hooge, F. N. 1/f Noise. Phys. B+C 1976, 83 (1), 14-23.

(3) Hooge, F. N. The Relation between 1/f Noise and Number of Electrons. Phys. B Phys. Condens. Matter 1990, 162 (3), 344-352.

(4) Tunaley, J. K. E. Conduction in a Random Lattice under a Potential Gradient. J. Appl. Phys. 1972, $43(11), 4783-4786$.

(5) Zhigal'skii, G. P. 1/ f Noise and Nonlinear Effects in Thin Metal Films. Physics-Uspekhi 1997, $40(6), 599-622$.

(6) Sharvin, Y. V. A Possible Method for Studying Fermi Surfaces. Sov. Phys. JETP 1965, 21 (3), 655-656. 
(7) Pud, S.; Kisner, A.; Heggen, M.; Belaineh, D.; Temirov, R.; Simon, U.; Offenhäusser, A.; Mourzina, Y.; Vitusevich, S. Features of Transport in Ultrathin Gold Nanowire Structures. Small 2013, 9 (6), 846-852.

(8) Handziuk, V.; Pud, S.; Coppola, M.; Kisner, A.; Vitusevich, S. Features of Noise in Ultrathin Gold Nanowire Structures. J. Stat. Mech. Theory Exp. 2016, $054023,54023$.

(9) Hooge, F. N.; Hoppenbrouwers, A. M. H. Contact Noise. Phys. Lett. A 1969, 29 (11), 642643.

(10) Hoppenbrouwers, A. M. H.; Hooge, F. N. 1/f Noise of Spreading Resistances. Philips Res. Reports 1970, 25 (2), 69-80.

(11) Hooge, F. N.; Hoppenbrouwers, A. M. H. 1/f Noise in Continuous Thin Gold Films. Physica 1969, 45, 386-392.

(12) Vandamme, L. K. J. 1/f Noise of Point Contacts Affected by Uniform Films. J. Appl. Phys. 1974, 45 (10), 4563-4565.

(13) Vandamme, L. K. J. Noise as a Diagnostic Tool for Quality and Reliability of Electronic Devices. Electron Devices, IEEE Trans. 1994, 41 (11), 2176-2187.

(14) Celasco, M.; Masoero, A.; Mazzetti, P.; Stepanescu, A. Electrical Conduction and Current Noise Mechanism in Discontinuous Metal Films. II. Experimental. Phys. Rev. B 1978, 17 (6), 2564-2574.

(15) Wu, Z.; Wu, S.; Oberholzer, S.; Steinacher, M.; Calame, M.; Schönenberger, C. Scaling of 1/f Noise in Tunable Break Junctions. Phys. Rev. B - Condens. Matter Mater. Phys. 2008, $78(23), 1-5$

(16) Ketchen, M. B.; Clarke, J. Temperature Fluctuations in Freely Suspended Tin Films at the Superconducting Transition. Phys. Rev. B 1978, 17 (1), 114-121.

(17) Schinabeck, C.; Härtle, R.; Weber, H. B.; Thoss, M. Current Noise in Single-Molecule 
Junctions Induced by Electronic- Vibrational Coupling. Phys. Rev. B - Condens. Matter Mater. Phys. 2014, 90 (7), 1-16.

(18) Brunner, J.; González, M. T.; Schönenberger, C.; Calame, M. Random Telegraph Signals in Molecular Junctions. J. Phys. Condens. Matter 2014, 26 (47), 474202.

(19) Xiang, D.; Sydoruk, V.; Vitusevich, S.; Petrychuk, M. V.; Offenhäusser, A.; Kochelap, V. A.; Belyaev, A. E.; Mayer, D. Noise Characterization of Metal-Single Molecule Contacts. Appl. Phys. Lett. 2015, 106 (6), 063702.

(20) Fakult, P.; Gr, L. Mechanical Controllable Break Junction in Liquid Environment: A Tool to Measure Single Molecules, 2005.

(21) Kim, Y.; Pietsch, T.; Erbe, A.; Belzig, W.; Scheer, E./Benzenedithiol: A Broad-Range Single-Channel Molecular Conductor. Nano Lett. 2011, 11 (9), 3734-3738.

(22) Tsutsui, M.; Teramae, Y.; Kurokawa, S.; Sakai, A. High-Conductance States of Single Benzenedithiol Molecules. Appl. Phys. Lett. 2006, 89 (16), 16-19.

(23) Vitusevich, S. A.; Danylyuk, S. V; Klein, N.; Petrychuk, M. V; Sokolov, V. N.; Kochelap, V. A.; Belyaev, A. E.; Tilak, V.; Smart, J.; Vertiatchikh, A.; et al. Excess Low-Frequency Noise in AlGaN / GaN-Based High-Electron-Mobility Transistors. Appl. Phys. Lett. 2002, $80(12), 2126$.

(24) Agraït, N.; Yeyati, A. L.; van Ruitenbeek, J. M. Quantum Properties of Atomic-Sized Conductors. Phys. Rep. 2003, 377 (2-3), 81-279.

(25) Adak, O.; Rosenthal, E.; Meisner, J.; Andrade, E. F.; Pasupathy, A. N.; Nuckolls, C.; Hybertsen, M. S.; Venkataraman, L. Flicker Noise as a Probe of Electronic Interaction at Metal-Single Molecule Interfaces. Nano Lett. 2015, 15 (6), 4143-4149.

(26) Vandamme, L. K. J. On the Calculation of 1/f Noise of Contacts. 1976, 96, 89-96.

(27) Coppus, G. W. M.; Vandamme, L. K. J. Spreading Resistance and 1/f Noise of Embedded 
Ellipsoidal Electrodes in a Conductor. Appl. Phys. 1979, No. 20, 119-123.

(28) Vandamme, L. K. J.; Groot, J. C. F. 1/f Noise and Resistance between Circular Electrodes. Electron Lett. 1978, 14 (2), 30-32.

(29) Vandamme, L. K. J.; de Kuijper, A. H. Conductance Noise Investigations on Symmetrical Planar Resistors with Finite Contacts. Solid State Electron. 1979, 22 (11), 981-986.

(30) Liang, C.; Vandamme, L. K. J.; Leroy, G.; Gest, J.; Liang, C.; Vandamme, L. K. J.; Leroy, G.; Resistance, J. G. Resistance and 1 / f Noise between Circular Contacts on Conductive Thin Films. 2012.

(31) Vandamme, L. K. J. Characterization of Contact Interface Film, Sheet Resistance and 1/f Noise with Circular Contacts. Fluct. Noise Lett. 2011, 10(04), 467-484.

(32) Ulbrich, W. Resistor Geometry Comparison with Respect to Current Noise and Trim Sensitivity. 1977, 4 (C), 63-68.

(33) Chen, T. M.; Rhee, J. G. The Effects of Trimming on the Current Noise of Thick Film Resistors. Solid State Technol. 1977, 49-53.

(34) Van Deursen, A. P. J.; Vandamme, L. K. J. Conductance Noise on a Square Sample, Calculation of the Geometrical Factor f(Q). Fluct. Noise Lett. 2007, 7 (2), L101-L110.

(35) Yoshida, H. The Effect of Resistor Geometry on Current Noise. IEEE Trans. Components, Hybrids, Manuf. Technot. 1993, 16 (3), 344-349.

(36) Hooge, F. N.; Kleinpenning, T. G. M.; Vandamme, L. K. J. Experimental Studies on 1 / f Noise. Reports Prog. Phys. 1981, 44, 479.

(37) Lukyanchikova, N. B. Noise Research in Semiconductor Physics; K., B. J., Ed.; Gordon and Breach Science Publishers: Amsterdam, 1997.

(38) Timsit, R. S. Electrical Conduction through Small Contact Spots. IEEE Trans. Components Packag. Technol. 2006, 29 (4), 727-734. 
(39) Xiang, D.; Zhang, Y.; Pyatkov, F.; Offenhäusser, A.; Mayer, D. Gap Size Dependent Transition from Direct Tunneling to Field Emission in Single Molecule Junctions. Chem. Commun. (Camb). 2011, 47 (16), 4760-4762.

(40) Jones, B. K.; Zhigal'skii, G. P. Physical Properties of Thin Metal Films, CRC Press.; 2003.

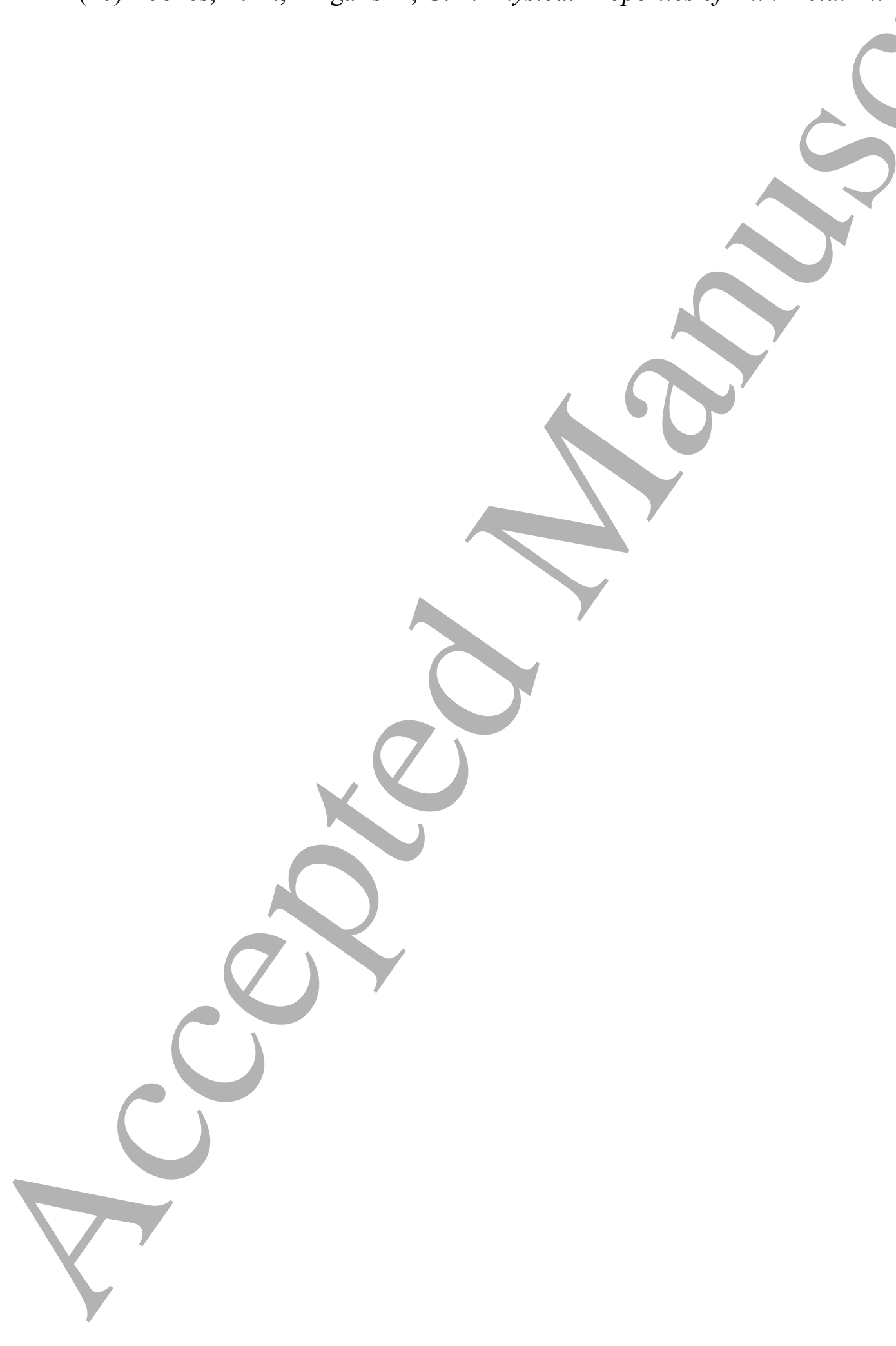

